\title{
A Comparison of Accelerated Life Testing Designs Within A Single Bayesian Inferential Framework
}

\author{
J. René van Dorp, D.Sc., The George Washington University \\ Thomas A. Mazzuchi, D Sc., The George Washington University \\ Jorge E. Garcidueñas, D.Sc., The George Washington University
}

Key Words: Accelerated Life Testing, Exponential Model, Test Design

\section{SUMMARY \& CONCLUSIONS}

Accelerated life testing (ALT) is the set of procedures used to reduce the time needed to obtain information related to life characteristics of an item, material or part of interest. Herein we focus on the comparison of different ALT designs (fixed stress, profile ALT, progressive step-stress ALT and regressive ALT) within a single Bayesian inference framework. We shall analyze the pre-posterior variance of the use-stress reliability based on a single failure over the course of the ALT for these different ALT designs. To the best of our knowledge Miller and Nelson in their 1983 paper entitled "Optimum Simple Step-Stress Plans for Accelerated Life Testing" in IEEE Trans. Reliability, Vol. R-32:59-65, were among the firsts to claim similarity of asymptotic estimator variance when comparing step-stress testing ALT to fixed stress ALT. The results in this paper shed some light on this claim within a Bayesian context.

\section{INTRODUCTION}

Accelerated life tests (ALT) are used to reduce the time needed to obtain information related to life characteristics of an item, material or part of interest. The two main avenues of research in ALT are: 1) the optimal design of the test and 2) the statistical inference from the failure data obtained. The importance of the design of these tests is paramount to the process of obtaining meaningful inferences about the life of the items in their use environment. Statistical inference is based on one of two paradigms, classical or Bayesian, and often makes use of a time transformation function which relates failure times at accelerated stress levels to failure times at use stress levels through the scale parameter of the life time distribution. Examples of such functions are the Power Law Model, the Eyring Model and the Arrhenius Model (see for example Mann, Schafer, and Singpurwalla [1]).

While there is a host of literature on ALT design, there has been a significant increase in the topic of step-stress accelerated life tests (SSALT) and their comparison to regular ALTs from a classical viewpoint (see for example, Miller and Nelson [2] and more recently Bai and Chung [3], Khamis and Higgins [4], and Khamis [5]). Bayesian approaches to ALT design have been considered in DeGroot and Goel [6], who investigated the optimal design of a partially accelerated life test (PALT), and Erkanli and Soyer [7] who develop a Bayesian decision theoretic method for selecting optimal fixed stress ALTs based on minimization of the expected predictive variance of the parameter of interest. To the best of our knowledge Miller and Nelson [2] were among the firsts to claim similarity of asymptotic estimator variance when comparing step-stress testing ALT to fixed stress ALT. To date, however, no one has considered a comparison of SSALTs and constant stress ALTs using a Bayesian approach. This is necessary, as in ALT the number of data items available is often not large, bringing into question the use of asymptotic values for comparison (which are basis for most classical design comparisons). This notion is well illustrated in McSorely, Lu, and Li [8].

In this paper we consider comparison of ALT designs using the Bayesian inference model developed in van Dorp and Mazzuchi [9]. The model assumes that the life length of the test item at any stress is exponentially distributed and that the failure rates of the life lengths are ordered with respect to the severity of the environment. This restricted ordering of the prior and posterior failure rate estimates acts as a nonparametric time transformation function. Two optimality criteria shall be used for ALT design comparisons: the usual pre-posterior variance and a measure which takes into account both pre-posterior mean and pre-posterior variance.

A general likelihood for the ALT design problem is presented in Section 2. In Section 3 the joint prior distribution of the parameters is introduced and in Section 4 expressions for posterior characteristics are developed. An example analysis is presented in Section 5.

\section{A GENERAL LIKELIHOOD FUNCTION FOR ACCELERATED LIFE TESTS}

Any statistical inference procedure involves developing a likelihood. The likelihood model developed in this section allows for a comprehensive representation of regular fixedstress, progressive step-stress, regressive step-stress and profile step-stress life testing (see for example Figure 1). Following the set up of van Dorp et. al [10], typically in ALT, an environment can be described by a collection of stress variables and their levels. In this model, it is assumed that a total of $K$ environments $E_{1}, \ldots, E_{K}$ are pre selected as candidate test environments within minimum and maximum 
design ranges of stresses to ensure that the predominant failure modes at use environments and accelerated environments are the same. It will be assumed that these candidate test environments may be rank ordered with respect to severity using engineering knowledge, i.e. $E_{1}\left(E_{K}\right)$ coincides with the least (most) severe environment.

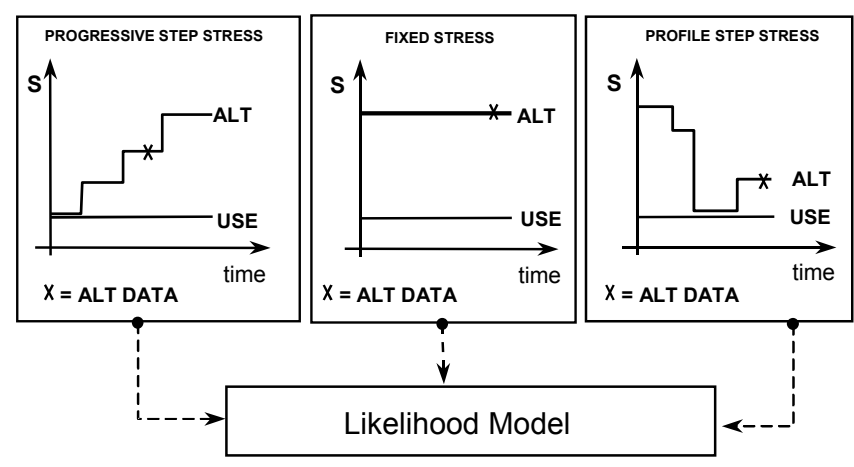

Fig. 1. Different ALT scenarios

The life time distribution in a constant stress environment $E_{e}$ will be modeled as an exponential life time distribution with failure rate $\lambda_{e}, e=1, \ldots, K$. The ordering of the test environments in terms of severity induces the same ordering in the associated failure rates, i.e.

$$
0 \equiv \lambda_{0}<\lambda_{1}<\cdots<\lambda_{K}<\lambda_{K+1} \equiv \infty .
$$

We shall consider a single test item subjected to an ALT with $m$ test intervals $\left[t_{i-1}, t_{i}\right) \quad i=1, \ldots, m$, with $t_{0} \equiv 0$, $t_{m+1} \equiv \infty$. If the item has not failed by time $t_{m}$, it is removed (censored) from testing. As testing may proceed in a variety of step patterns, the actual test environment in $\left[t_{i-1}, t_{i}\right)$ will be denoted by $E_{a_{i}}, a_{i} \in\{1, \ldots, K\}$. With the above setup, we have for the ALT reliability function during the test at time $t \in\left[t_{i-1}, t_{i}\right)$ Eq. (2) and for the ALT failure density or likelihood of a failure at time $t$ during the test Eq. (3).

$$
\begin{aligned}
& R\left(t \mid \lambda_{1}, \ldots, \lambda_{K}\right)= \\
& \exp \left\{-\lambda_{a_{1}} t\right\} \quad t \in\left[0, t_{1}\right) \\
& \left\{\exp \left\{\begin{array}{c}
-\sum_{j=1}^{i-1} \lambda_{a_{j}}\left(t_{j}-t_{j-1}\right) \\
-\lambda_{a_{i}}\left(t-t_{i-1}\right)
\end{array}\right\} t \in\left[t_{i-1}, t_{i}\right)\right. \\
& \exp \left\{-\sum_{j=1}^{m} \lambda_{a_{j}}\left(t_{j}-t_{j-1}\right)\right\} \quad t>t_{m} \\
& f\left(t \mid \lambda_{1}, \ldots, \lambda_{K}\right)=-\frac{d}{d t} R\left(t \mid \lambda_{1}, \ldots, \lambda_{K}\right) \\
& = \begin{cases}\lambda_{a_{1}} R\left(t \mid \lambda_{1}, \ldots, \lambda_{K}\right) & t \in\left[0, t_{1}\right) \\
\lambda_{a_{i}} R\left(t \mid \lambda_{1}, \ldots, \lambda_{K}\right) & t \in\left[t_{i-1}, t_{i}\right) \\
0 & t>t_{m}\end{cases}
\end{aligned}
$$

Figure 2 presents a comparison of the ALT reliability and density functions for a fixed stress and profile ALT test. The solid lines in Figures 2A and 2B (denoted 213) display the ALT reliability and density functions for a profile ALT test as defined by Eq. (4).

$$
\begin{gathered}
a_{1}=2, a_{2}=1, a_{3}=3, \\
t_{1}=1, t_{2}=2, t_{3}=3, \\
\lambda_{1}=\frac{1}{2}, \lambda_{2}=1, \lambda_{3}=1 \frac{1}{2} .
\end{gathered}
$$

From (2) and the unit step lengths we obtain for the reliability at the end of the ALT test (i.e. the survival probability)

$$
R(3)=\exp \left(-\sum_{i=1}^{3} \lambda_{a_{i}}\right)=\exp (-3) \approx 0.05
$$

for both ALT setups in Figure 2. Hence, both ALT setups in Figure 2 are comparable from this vantage point. The immediate question arises whether there is any benefit to profile stepping (or step stress testing in point. profile stepping (or step stress testing in general) compared to a fixed stress ALT setup. In this paper we shall compare different ALT step patterns by sampling different values of $\lambda_{i}, i=1, \ldots, 3$ from a prior distribution to be defined in the next section. For consistency in comparison, we shall evaluate posterior use stress statistics keeping the ALT survival probability constant under different values of $\lambda_{i}$. This is achieved by "stretching" or "shrinking" the total test time $t_{m}$ keeping the length of each test interval the same.

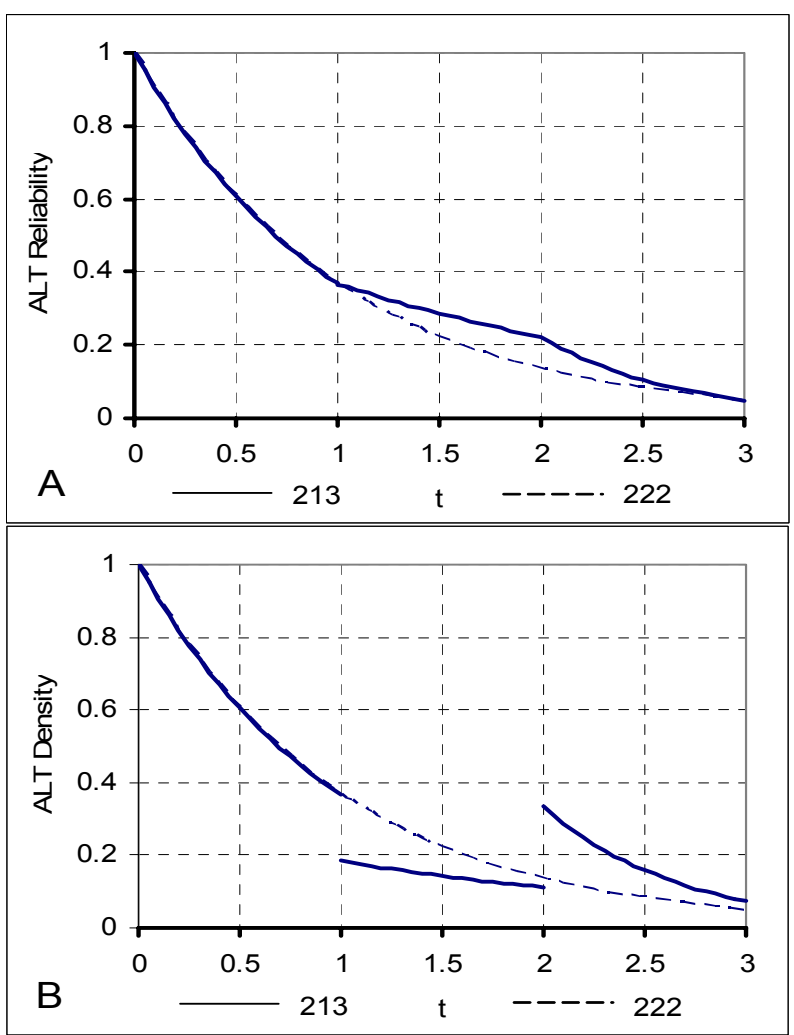

Fig. 2. ALT reliability function and ALT density function for a profile ALT test defined by (4) as a function of time $t$.

Introducing the transformation

$$
\lambda_{e}=-\operatorname{Ln}\left(u_{e}\right) \Leftrightarrow u_{e}=\exp \left(-\lambda_{e}\right),
$$


(2) and (3) may be expressed in terms of $u_{e}$ for mathematical convenience (see Section 3). Quantities $u_{e}$ may be interpreted as the reliability after 1 time unit of exposure to environment $e$ and will henceforth be referred to as unit reliabilities in the different testing environments. Substituting (6) into (2) (and (3)), links the reliability of the test item at time $t$ (the likelihood of failure at time $t$ ) to the unit reliabilities $u_{e}, e=$ $1, \ldots, K$. We have respectively:

$$
\begin{gathered}
R\left(t \mid u_{1}, . ., u_{K}\right)= \begin{cases}\left(u_{a_{i}}\right)^{t} & t \in\left[0, t_{1}\right) \\
\left\{\prod_{j=1}^{i-1}\left(u_{a_{j}}\right)^{t_{j}-t_{j-1}}\right\}\left(u_{a_{i}}\right)^{t-t_{i-1}} & t \in\left[t_{i-1}, t_{i}\right) \\
\prod_{j=1}^{m}\left(u_{a_{j}}\right)^{t_{j}-t_{j-1}} & t>t_{m}\end{cases} \\
f\left(t \mid u_{1}, \ldots, u_{K}\right)= \begin{cases}-\operatorname{Ln}\left(u_{a_{i}}\right) R\left(t \mid \lambda_{1}, \ldots, \lambda_{K}\right) & t \in\left[0, t_{1}\right), \\
0 & i=1, \ldots, m\end{cases}
\end{gathered}
$$

The mathematical forms of expressions (7) and (8) are more conducive to posterior updating as compared to expressions (2) and (3) utilizing the prior distribution to be defined in the next section.

\section{PRIOR DISTRIBUTION}

Denoting $\Lambda_{e}$ to be the random variable associated with failure rate realization $\lambda_{e}$ and using (6) and (1) it follows that

$$
0 \equiv u_{K+1}<U_{K}<\cdots<U_{1}<u_{0} \equiv 1 .
$$

Rather than defining a prior distribution for $\underline{\Lambda}=\left(\Lambda_{1}, \ldots, \Lambda_{K}\right)$ exhibiting property (1) for all realizations, $\underline{\lambda}=\left(\lambda_{1}, \ldots, \lambda_{K}\right)$, one may equivalently define a prior for $\underline{U}=\left(U_{1}, \ldots, U_{K}\right)$ exhibiting property (9). Concentrating on $\underline{U}=\left(U_{1}, \ldots, U_{K}\right)$ a prior distribution which is: i) mathematically tractable, ii) is defined over the region specified in (9), and iii) imposes no other restrictions on $\underline{u}$ is the multivariate Ordered Dirichlet distribution,

$$
\Pi\{\underline{u} \mid \eta, \underline{v}\}=\frac{\prod_{e=1}^{K+1}\left(u_{e-1}-u_{e}\right)^{\eta \cdot v_{e}-1}}{\mathbb{D}(\eta, \underline{v})}
$$

where, $\eta>0, v_{e}>0, e=1, \ldots, K+1, \sum_{i=1}^{K+1} v_{i}=1$,

$$
\mathbb{D}(\eta, \underline{v})=\left\{\prod_{i=1}^{K+1} \Gamma\left(\eta v_{e}\right)\right\} / \Gamma(\eta)
$$

and $\Gamma(\bullet)$ is the gamma function satisfying $\Gamma(n+1)=n \Gamma(n)$.

Using the transformation given by the RHS of (6) allows one to take full advantage of the mathematical properties of the Ordered Dirichlet Distribution. For a detailed discussion of these properties see Van Dorp and Mazzuchi [9]. The joint moment expression for $\underline{U} \sim \Pi\{\underline{u} \mid \eta, \underline{v}\}$ defined by (10) follows as:

$$
E_{\underline{U}}\left[\prod_{e=1}^{K}\left(U_{e}\right)^{k_{e}} \mid \eta, \underline{v}\right]=\frac{\Gamma(\eta)}{\Gamma\left(\eta v_{K+1}\right)} \prod_{e=1}^{K} \frac{\Gamma\left(\eta v_{\geq e+1}+k_{\geq e}\right)}{\Gamma\left(\eta v_{\geq e}+k_{\geq e}\right)}
$$

where

$$
v_{\geq e+1}=\sum_{i=e+1}^{K+1} v_{i} \text { and } k_{\geq e}=\sum_{i=e}^{K} k_{i}
$$

We have added the subscript $\underline{U}=\left(U_{1}, \ldots, U_{K}\right)$ to emphasize that the expectation in (12) is taken with respect to the prior distribution (10)

\section{POSTERIOR MOMENTS AFTER A SINGLE TEST ITEM}

In this section we shall derive closed form expression for posterior use stress moments of order $k$ for $k \geq 1$, given the failure or survival of the ALT test item up to time $t$. These derivations employ (a) the joint moments expressions (12), (b) the mathematical form of $R\left(t \mid u_{1}, \ldots, u_{K}\right)$ defined by (7), (c) the fact that

$$
\begin{gathered}
\int_{0}^{1} \int_{0}^{u_{1}} \cdots \int_{0}^{u_{K-1}} u_{1}^{m} f\left(t \mid u_{1}, \ldots, u_{K}\right) \times \Pi\{\underline{u} \mid \eta, \underline{v}\} d u_{K} \cdots d u_{1} \\
=-\frac{d}{d t} \int_{0}^{1} \int_{0}^{u_{1}} \cdots \int_{0}^{u_{K-1}} u_{1}^{m} R\left(t \mid u_{1}, \ldots, u_{K}\right) \times \\
\Pi\{\underline{u} \mid \eta, \underline{v}\} d u_{K} \cdots d u_{1}
\end{gathered}
$$

and (d) that for $W(t)=\prod_{e=1}^{K}\left\{g_{e}(t) / h_{e}(t)\right\}$ we have

$$
\frac{d}{d t} W(t)=W(t) \times \sum_{e=1}^{K}\left[\frac{\frac{d}{d t} g_{e}(t)}{g_{e}(t)}-\frac{\frac{d}{d t} h_{e}(t)}{h_{e}(t)}\right]
$$

\subsection{Posterior moments after test a item survived up to time $t$}

Applying Bayes theorem allows for derivation of closed form posterior moments of the unit reliability in the use stress failure rate environment, i.e. $u_{1}$. We have

$$
\begin{gathered}
E_{\underline{U}}\left[U_{1}^{m} \mid \eta, \underline{v}, t\right]=\int_{0}^{1} \int_{0}^{u_{1}} \cdots \int_{0}^{u_{K-1}} u_{1}^{m} \Pi\{\underline{u} \mid t, \eta, \underline{v}\} d u_{K} \cdots d u_{1} \\
=\frac{\int_{0}^{1} \cdots \int_{0}^{u_{K-1}} u_{1}^{m} R\left(t \mid u_{1}, \ldots, u_{K}\right) \Pi\{\underline{u} \mid \eta, \underline{v}\} d u_{K} \cdots d u_{1}}{\int_{0}^{1} \cdots \int_{0}^{u_{K-1}} R\left(t \mid u_{1}, \ldots, u_{K}\right) \Pi\{\underline{u} \mid \eta, \underline{v}\} d u_{K} \cdots d u_{1}}
\end{gathered}
$$

Denoting the amount of time that the test item has been exposed to environment $E_{e}$ prior to $t$ with $\tau_{e}(t)$, one obtains

$$
\begin{array}{r}
\tau_{e}(t)=\sum_{j=1}^{m}\left(t_{j}-t_{j-1}\right) 1_{a_{j}}(e) \times 1_{[0, t)}\left(t_{j}\right)+ \\
\sum_{j=1}^{m}\left(t-t_{j-1}\right) 1_{a_{j}}(e) \times 1_{\left[t_{j-1}, t_{j}\right)}(t),
\end{array}
$$

where

$$
1_{a_{j}}(e)=
$$

$\begin{cases}1 & \text { if in test interval } j \text { the test environment } a_{j} \text { is } e \\ 0 & \text { else, }\end{cases}$

and

$$
1_{[0, t)}\left(t_{j}\right)= \begin{cases}1 & t_{j} \in[0, t) \\ 0 & \text { else. }\end{cases}
$$

Employing the mathematical definition of $R\left(t \mid u_{1}, \ldots, u_{K}\right)$ (7): 


$$
\begin{gathered}
\int_{0}^{1} \int_{0}^{u_{1}} \cdots \int_{0}^{u_{K-1}} u_{1}^{m} R\left(t \mid u_{1}, \ldots, u_{K}\right) \times \Pi\{\underline{u} \mid \eta, \underline{v}\} d u_{K} \cdots d u_{1} \\
=E_{\underline{U}}\left[\prod_{e=1}^{K}\left(U_{e}\right)^{k_{e}(m, t)}\right]
\end{gathered}
$$

where

$$
k_{e}(m, t)= \begin{cases}\tau_{e}(t)+m & e=1 \\ \tau_{e}(t) & e>1\end{cases}
$$

Substitution of (20) into (16) yield

$$
E_{\underline{U}}\left[U_{1}^{m} \mid \eta, \underline{v}, t\right]=\frac{E_{\underline{U}}\left[\prod_{e=1}^{K}\left(U_{e}\right)^{k_{e}(m, t)} \mid \eta, \underline{v}\right]}{E_{\underline{U}}\left[\prod_{e=1}^{K}\left(U_{e}\right)^{k_{e}(0, t)} \mid \eta, \underline{v}\right]}
$$

where $k_{e}(m, t)$ is defined by (21), $\tau_{e}(t)$ by (17) and the prior joint moments are defined by (12).

\subsection{Posterior moments after a test item failed at time $t$}

Analogously to (16) and employing (14) and (20) it immediately follows that

$$
\begin{aligned}
& E_{\underline{U}}\left[U_{1}^{m} \mid \eta, \underline{v}, t\right]= \\
& \frac{\int_{0}^{1} \cdots \int_{0}^{u_{K-1}} u_{1}^{m} f\left(t \mid u_{1}, \ldots, u_{K}\right) \Pi\{\underline{u} \mid \eta, \underline{v}\} d u_{K} \cdots d u_{1}}{\int_{0}^{1} \cdots \int_{0}^{u_{K-1}} f\left(t \mid u_{1}, \ldots, u_{K}\right) \Pi\{\underline{u} \mid \eta, \underline{v}\} d u_{K} \cdots d u_{1}}= \\
& \frac{d}{d t} \int_{0}^{1} \cdots \int_{0}^{u_{K-1}} u_{1}^{m} R\left(t \mid u_{1}, \ldots, u_{K}\right) \Pi\{\underline{u} \mid \eta, \underline{v}\} d u_{K} \cdots d u_{1} \\
& \frac{d}{d t} \int_{0}^{1} \cdots \int_{0}^{u_{K-1}} R\left(t \mid u_{1}, \ldots, u_{K}\right) \Pi\{\underline{u} \mid \eta, \underline{v}\} d u_{K} \cdots d u_{1} \\
& \frac{d}{d t} E_{\underline{U}}\left[\prod_{e=1}^{K}\left(u_{e}\right)^{k_{e}(m, t)} \mid \eta, \underline{v}\right] / \frac{d}{d t} E_{\underline{U}}\left[\prod_{e=1}^{K}\left(u_{e}\right)^{k_{e}(0, t)} \mid \eta, \underline{v}\right]
\end{aligned}
$$

where $k_{e}(m, t)$ is defined by (21) and $\tau_{e}(t)$ by (17). Utilizing (15) we immediately obtain

$$
\begin{aligned}
& \frac{d}{d t} E_{\underline{U}}\left[\prod_{e=1}^{K}\left(U_{e}\right)^{k_{e}(m, t)} \mid \eta, \underline{v}\right]=E_{\underline{U}}\left[\prod_{e=1}^{K}\left(U_{e}\right)^{k_{e}(m, t)} \mid \eta, \underline{v}\right] \\
& \times \sum_{e=1}^{K}\left[\frac{\frac{d}{d t} \Gamma\left\{\beta v_{\geq e+1}+k_{\geq e}(m, t)\right\}}{\Gamma\left\{\beta v_{\geq e+1}+k_{\geq e}(m, t)\right\}}-\frac{\frac{d}{d t} \Gamma\left\{\beta v_{\geq e}+k_{\geq e}(m, t)\right\}}{\Gamma\left\{\beta v_{\geq e}+k_{\geq e}(m, t)\right\}}\right]
\end{aligned}
$$

where

$$
k_{\geq e}(m, t)=\sum_{i=e}^{K} k_{i}(m, t)
$$

To further reduce (24) we note that $\frac{d}{d t} \Gamma(\mathrm{t}) / \Gamma(\mathrm{t})$ may be recognized as the psi or digamma function $\psi(\cdot)$ and thus

$$
\begin{aligned}
& \frac{\frac{d}{d t} \Gamma\left\{\beta v_{\geq e+1}+k_{\geq e}(m, t)\right\}}{\Gamma\left\{\beta v_{\geq e+1}+k_{\geq e}(m, t)\right\}}= \\
& \Psi\left\{\beta v_{\geq e+1}+k_{\geq e}(m, t)\right\} \sum_{i=e}^{K} 1_{a_{j}}(i) \times 1_{\left[t_{i-1}, t_{i}\right)}(t),
\end{aligned}
$$

since

$$
\frac{d}{d t}\left\{\beta v_{\geq e+1}+k_{\geq e}(m, t)\right\}=\sum_{i=e}^{K} \frac{d}{d t} k_{i}(m, t)
$$

and finally

$$
\frac{d}{d t} k_{i}(m, t)=\frac{d}{d t} \tau_{i}(t)=1_{a_{j}}(i) \times 1_{\left[t_{j-1}, t_{j}\right)}(t) .
$$

\subsection{Example}

Let us consider the prior distribution on the unit reliabilities $\underline{U}$ with prior parameters

$$
\begin{aligned}
& \eta=5, v_{1}=0.39347, v_{2}=0.23865, \\
& v_{3}=0.14475, v_{4}=0.22313
\end{aligned}
$$

These prior parameters settings are chosen for illustration purposes such that:

$$
E_{\underline{U}}\left[U_{e} \mid \eta, v_{\geq e+1}\right]=\exp \left(-\lambda_{e}\right),
$$

$$
\text { where } \lambda_{1}=\frac{1}{2}, \lambda_{2}=1, \lambda_{3}=1 \frac{1}{2} \text {. }
$$

These values coincide with the ALT setup in Figure 2. Figure 3 depicts the behavior of $E_{\underline{U}}\left[U_{1} \mid \eta, \underline{v}, t\right]$ given by (23) for $t<$ 3 (i.e. the test item failed before the end of the ALT test) and $E_{U}\left[U_{1} \mid \eta, \underline{v}, t\right]$ given by (22) for $t>3$ (i.e. the test item survived the ALT test) for both the profile 213 ALT set-up and the fixed stress ALT setup such that $\lambda_{a_{i}}=2, i=1,2,3$ (indicated by 222 in Figure 3). Note that the jumps up and down of the solid line in Figure 3 mimic those of the ALT density function $f\left(t \mid \lambda_{1}, \lambda_{2}, \lambda_{3}\right) \equiv f\left(t \mid u_{1}, u_{2}, u_{3}\right)$ in Figure $2 \mathrm{~B}$. The horizontal dotted line depicts the average value of the prior use stress unit reliability i.e.

$$
E_{\underline{U}}\left[U_{1} \mid \eta, v_{\geq 2}\right]=\exp \left(-\lambda_{1}\right)=\exp \left(-\frac{1}{2}\right) \approx 0.6065
$$

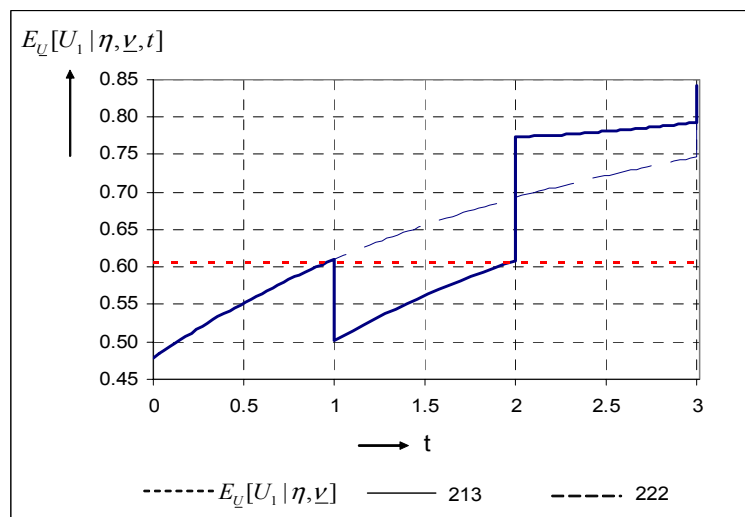

Fig. 3. Posterior expected use stress reliability as a function of test failure time and total test time 3 for prior parameter setting (29) and the ALT density functions in Figure 2B.

\section{A COMPARISON OF ALT STEP PATTERNS}

In this section we shall first set the stage of what is called a "pre-posterior analysis" of ALT step patterns in Section 5.2, by providing in Section 5.1 a preliminary comparison of the ALT profile and ALT fixed stress test presented in Figure 2. We utilize this example to motivate a single measure to 
compare different step patterns that rewards both calibration and precision.

5.1. A preliminary comparison of the ALT setups in Figure 2 Due to the manner (30) in which the prior parameters (29) are chosen, it seems intuitive to expect that given a random failure time $T_{\lambda}{ }^{*}$, where $T_{\lambda}{ }^{*}$ follows either of the ALT step patterns 213 and 222 defined in Figure $2 B$ with $\underline{\lambda}^{*}=(1 / 2,1$, $1-1 / 2$ ), average posterior unit reliability should be equal or close to prior unit reliability, i.e.

$$
E_{T_{\underline{\underline{*}}^{*}}}\left[E_{\underline{U}}\left[U_{1} \mid \eta, \underline{v}, T_{\underline{\lambda}^{*}}\right]\right] \approx E_{\underline{U}}\left[U_{1} \mid \eta, v_{\geq 2}\right]
$$

where the value of $E_{\underline{U}}\left[U_{1} \mid \eta, v_{\geq 2}\right]$ is given by (31). (It is important here to emphasize the distinction between $E_{\underline{U}}\left[U_{1} \mid \eta, \underline{v}, T_{\lambda}^{*}\right]$ - which is a posterior mean and $E_{\underline{U}}\left[U_{1}, T_{\lambda}{ }^{*} \mid \eta, \underline{v}\right]$ - which is a joint mean. If we were to substitute $E_{\underline{U}}\left[U_{1}, T_{\lambda}{ }^{*} \mid \eta, \underline{v}\right]$ for $E_{\underline{U}}\left[U_{1} \mid \eta, \underline{v}, T_{\lambda}{ }^{*}\right]$ in (32) equality would hold due to the law of total probability.) An additional measure to monitor agreement between the prior and the posterior distribution is the posterior variance. An increase in posterior variance compared to prior variance is typically associated in a Bayesian analysis with a disagreement between prior information and the observed data. To test assertion (32) and observe prior-posterior disagreement, we generate samples $t_{i}^{0}, i=1, \ldots, 5000$ from both ALT density functions in Figure $2 \mathrm{~B}$ and evaluate:

$$
\begin{array}{r}
\hat{E}_{T_{\lambda^{*}}}\left[E_{\underline{U}}\left[U_{1} \mid \eta, \underline{v}, T_{\underline{\lambda}^{*}}\right]\right]=\frac{1}{5000} \sum_{i=1}^{5000} E_{\underline{U}}\left[U_{1} \mid \eta, \underline{v}, t_{i}^{0}\right] \\
\hat{E}_{T_{\underline{\lambda}^{*}}}\left[\operatorname{Var}_{\underline{U}}\left[U_{1} \mid \eta, \underline{v}, T_{\underline{\lambda^{*}}}\right]\right]=\frac{1}{4999} \sum_{i=1}^{4999} \operatorname{Var}_{\underline{U}}\left[U_{1} \mid \eta, \underline{v}, t_{i}^{0}\right]
\end{array}
$$

The results are presented in Table 1 as well as the prior mean and variance $E_{\underline{U}}\left[U_{1} \mid \eta, v_{\geq 2}\right], \operatorname{Var}_{\underline{U}}\left[U_{1} \mid \eta, v_{\geq 2}\right]$ of use stress unit reliability.

Table 1. A preliminary comparison of the ALT step patterns in Fig. 2 utilizing prior parameter values $\eta, \underline{v}$ provided in (29).

\begin{tabular}{lcc}
\hline & ALT 213 & ALT 222 \\
\hline$E_{\underline{U}}\left[U_{1} \mid \eta, v_{\geq 2}\right]$ & 0.6065 & 0.6065 \\
\hline$\hat{E}_{T_{\underline{\lambda}^{*}}}\left[E_{\underline{U}}\left[u_{1} \mid \eta, \underline{v}, T_{\underline{\lambda}^{*}}\right]\right]$ & 0.6025 & 0.6024 \\
\hline $\operatorname{Var}_{\underline{U}}\left[U_{1} \mid \eta, v_{\geq 2}\right]$ & & \\
\hline$\hat{E}_{T_{\underline{\lambda}^{*}}}\left[\operatorname{Var}_{\underline{U}}\left[u_{1} \mid \eta, \underline{v}, T_{\underline{\lambda}^{*}}\right]\right]$ & 0.0795 & 0.0795 \\
\hline
\end{tabular}

Note that the results for the ALT 213 profile stress test indicate slightly better calibration (since its values in the second column is closer to the one in the first column compared to the ALT 222 set-up) and higher precision (since the variance in its third column is less than that of the ALT 222 set-up). Both ALT scenarios indicate agreement between prior distribution and observe data (on average) since the fourth column entries are reduced by more than $50 \%$ of the third column entries.
A test evaluation measure that rewards both calibration and precision may be defined to be

$$
\begin{aligned}
& \mathcal{S}\left(T_{\underline{\lambda^{*}}}, \eta, \underline{v}\right)=\left[\left\{\hat{E}_{T_{\underline{\lambda^{*}}}}\left[E_{\underline{U}}\left[u_{1} \mid \eta, \underline{v}, T_{\underline{\lambda}^{*}}\right]\right]-E_{\underline{U}}\left[U_{1} \mid \eta, v_{\geq 2}\right]\right\}^{2}\right. \\
& \left.+\hat{E}_{{\underline{\lambda^{*}}}^{*}}\left[\operatorname{Var}_{\underline{U}}\left[u_{1} \mid \eta, \underline{v}, T_{\underline{\lambda}^{*}}\right]\right]\right]^{-\frac{1}{2}}
\end{aligned}
$$

The second term (first term) in (35) addresses precision (calibration). Recalling that precision is defined as the reciprocal of the variance it follows that higher values of $\mathcal{S}\left(T_{\lambda}{ }^{*}, \eta, \underline{v}\right)$ are preferred over lower values. For the results in Table 1 we have

$$
\mathcal{S}_{213}\left(T_{\underline{\lambda}^{*}}, \eta, \underline{v}\right) \approx 5.31 \text { and } \mathcal{S}_{222}\left(T_{\underline{\lambda}^{*}}, \eta, \underline{v}\right) \approx 5.28,
$$

and based on the evaluation measure defined by (36) we prefer the profile step stress over the fixed stress one.

However, the comparison in (36) can only be considered a preliminary one (and not a meaningful one from Bayesian perspective) since it assumed perfect knowledge about failure rates $\underline{\lambda}^{*}=(1 / 2,1,1-1 / 2)$ (and thus indirectly also about unit reliabilities $\underline{u})$.

\subsection{A comparison analysis of step patterns in ALT}

Instead of assuming a fixed value $\underline{\lambda}^{*}=(1 / 2,1,1-1 / 2)$ we shall sample failure rate vectors $\underline{\lambda}^{i}, i=1, \ldots, 1000$ indirectly, by sampling vectors of unit reliabilities $\underline{u}^{i}, i=1, \ldots, 1000$ from the prior distribution (10) with the same prior parameters (29) and utilizing the transformations (6). For each realization $\underline{\lambda}^{i}$, we "stretch" the length of time $t_{m}$ of the ALT test, to ensure the same ALT survival probability of $\approx 0.05$ (see (5)) of the ALT densities in Figure 2B (which terminate at $t_{m}=3$ ). Next, we sample a 1000 failure times of the random variable $T_{\lambda} i$ with a predefined ALT step-pattern and evaluate one_realization of the performance measures

$$
\begin{gathered}
\hat{E}_{T_{\underline{\lambda^{i}}}}\left[E_{\underline{U}}\left[U_{1} \mid \eta, \underline{v}, T_{{\underline{\lambda^{i}}}^{i}}\right]\right], \hat{E}_{T_{\underline{\lambda^{i}}}}\left[\operatorname{Var}_{\underline{U}}\left[U_{1} \mid \eta, \underline{v}, T_{\underline{\lambda}^{i}}\right]\right] \\
\text { and } \mathcal{S}\left(T_{\underline{\lambda^{i}}}, \eta, \underline{v}\right)
\end{gathered}
$$

defined by (33), (34) and (35). (We reduce the sample size from $T_{\lambda} i$ from 5000 to 1000 to reduce calculation time.) Summarizing, we shall obtain a sample of 1000 values of the statistics indicated by (37) associated with a predefined steppattern. Figure 4 summarizes the algorithm to generate these observations.

Figure 5 summarizes the analysis results for two fixed stress ALT's (indicated by 111 and 222), a progressive ALT (indicated by 123) and a profile ALT indicated by 213). The horizontal lines on each sub-plot indicate the prior values of the performance measure $\mathcal{S}\left(T_{\lambda}{ }^{*}, \eta, \underline{v}\right)$, whereas the values indicate the average value for the iteration series plotted in each subfigure. By comparing Figures A.111 and B.222 we immediately observe a larger sensitivity in the iteration series $i=1, \ldots, 1000$ to failure time data obtained in the use stress environment 1 as compared to the accelerated Environment 2. 


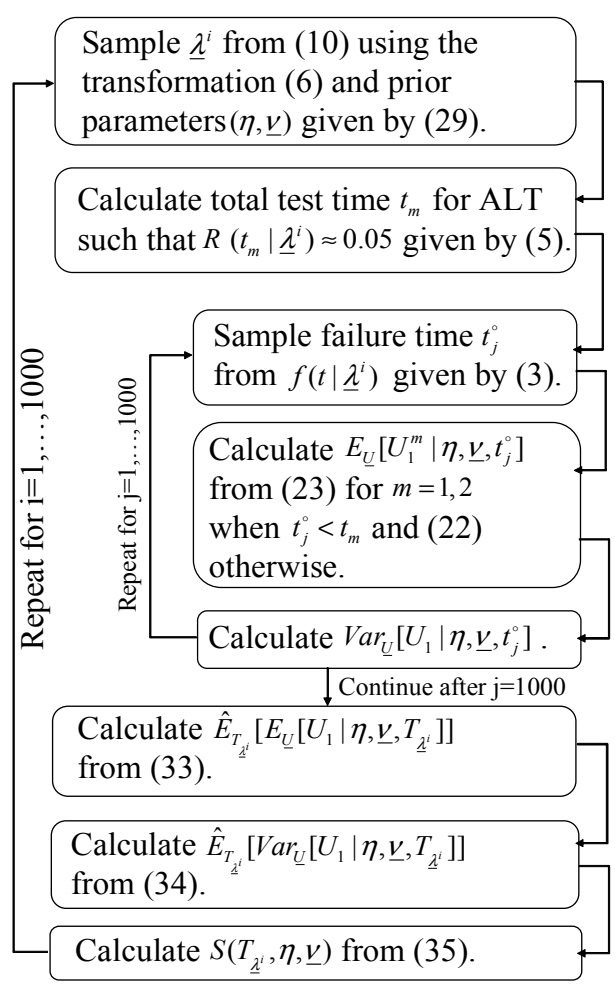

Fig. 4. Pre-posterior analysis algorithm to obtain 1000 values of the test statistics define by (37)

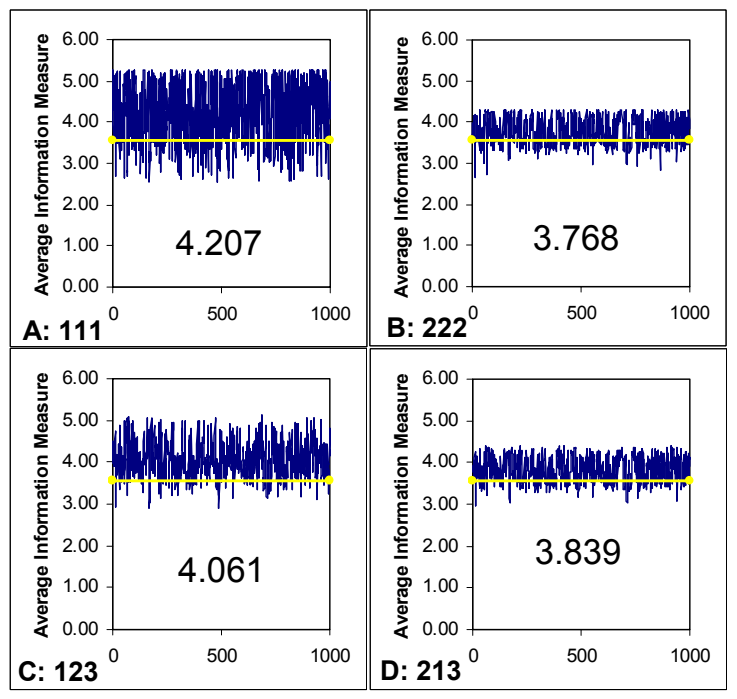

Fig. 5. Pre-posterior analysis of different ALT test patterns

This larger sensitivity can be explained immediately by the correlations $\rho_{e f}$ between unit reliabilities $U_{e}$ and $U_{f}$ defined by (12). We obtain from the prior parameters (29):

$$
\rho_{12} \approx 0.61 ; \rho_{13} \approx 0.43 ; \rho_{23}=0.70
$$

The correlation $\rho_{12} \approx 0.61$ in (38) thus acts as a "dampening" effect of data observed in the environment on posterior updating of use stress unit reliability in environment 1 . Figure A:111 with the higher evaluation score 4.207 for $\mathcal{S}\left(T_{\lambda}{ }^{*}, \eta, \underline{v}\right)$ (see Eq. (35)), shows a preference for obtaining data in the use stress environment than in the higher stress environment 2. (Again, this also follows immediately from the correlation coefficient $\rho_{13} \approx 0.61$ in (38)). Keeping ALT survivability constant however, implies excessive test time at the use stress levels, which is why ALT is being considered in the first place. On the other hand, while testing in a higher stress environment reduces test time, more test results may be needed to confirm or contest specified prior use stress parameters. Differences observed above are similar but exacerbated for a fixed stress ALT at environment 3 (but are not displayed) due to the lower correlation $\rho_{12} \approx 0.43$.

Comparing the progressive step-stress results in Figures C:123 with those in their "111 and 222" counterparts, it is interesting to note a lesser sensitivity in prior updating than observed in the "111" figures, but a slightly larger one than observed in the "222" figures. Similar comparisons of the figure associated with the profile ALT set-up 213 reveal an improvement of results when compared to fixed stress testing at environment 2, but less favorable results when compared to the progressive ALT 123 set-up. (Results associated with other profiles are not displayed here, but are worse than the "213" ones and are available upon request).

Summarizing our results seem to confirm within a Bayesian paradigm a favorable conclusion earlier made by Miller and Nelson [2] in a classical paradigm towards progressive ALT step-stress testing compared to fixed stress ALT. Moreover, our results seem to indicate a preference from an inference point of view toward progressive step-stress testing when compared to any other profile or regressive step patterns.

\section{A CONCLUDING REMARK}

It is important to recall here that comparisons in Section 5 are conducted in a context where the ALT survival probability is fixed at approximately $5 \%$ regardless of the step-patterns and values of the sample failure rates $\underline{\lambda}^{i}$ (which requires "stretching" or "shrinking" the total test time). The comparison conclusion between fixed stress testing at use stress and at a higher stress level may be thus be considered "kicking in an open door". Of course, we would prefer to test in use stress than in a higher stress from an inference point of view if we had the time to do so. The important observation is, however, that the larger the difference between the higher stress environment and the use stress environment, the larger the "dampening effect" of high stress environment data on use stress updating (due to an ever smaller correlation between the two). Consequently, a larger amount of data at higher stress levels may be needed to observe similar departures from specified prior use stress information when compared to use stress testing. In our judgment, this dampening effect is intuitive and realistic.

Such a dampening effect is not present when one utilizes a one-to-one transformations function (such as for example the power law) between a higher stress environments failure rate and the use stress one. Essentially, the one-to-one time transformation function "translates" a failure in a high stress environment to one in use stress (and thus assumes no loss of 
information by testing at a higher stress level). "Correctness" of any statistical inference procedure that utilizes such a oneto-one time transformation function therefore "stands and falls" with the correctness of the time transformation function used. We are of the opinion that neither the functional form of the transformation function can be known exactly, nor its parameters (especially when more than one stress parameter is varied at one time), which is why we propose instead the use of a relaxed ranking assumption of environments in terms of failure rates (see (1)) and a subsequent Bayesian analysis as presented in this paper.

\section{ACKNOWLEDGEMENTS}

We are thankful to the session chair Duane Dietrich, the referee Thomas Hassett and the editor Joel Nachlas. Their devotion, far beyond the call of duty, and valuable comments improved the contents and presentation of this paper.

\section{REFERENCES}

1. N.R. Mann, R.E. Schafer, and N.D. Singpurwalla, Methods for Statistical Analysis of Reliability and Life Data, John Wiley \& Sons, 1974.

2. R.W. Miller and W.B. Nelson, "Optimum simple stepstress plans for accelerated life testing," IEEE Transactions on Reliability, R-32 (1), 1983, pp 59-65.

3. D.S. Bai and S.W. Chung, "Optimal design of partially accelerated life tests for the exponential distribution under type-I censoring," , 41 IEEE Transactions on Reliability (3), 1992, pp 400-406.

4. I.H. Khamis and J.J. Higgins, "Optimum 3-Step StepStress Tests." , 45 (2), IEEE Transactions on Reliability 1996, pp 341-345.

5. I.H. Khamis, "Comparison between constant and stepstress tests for Weibull models," International Journal of Quality \& Reliability Management, 14 (1), 1997, pp 7481.

6. M.H. DeGroot and P.K. Goel, "Bayesian estimation and optimal designs in partially accelerated life testing," Naval Research Logistics Quarterly, 26, 1979, pp $223-$ 235.

7. A. Erkanli and R. Soyer, "Simulation-based designs for accelerated life tests," Journal of Statistical Planning and Inference, 90 (2), 2000, pp 335-348.

8. E.O. McSorley, J.C. Lu and C.S. Li, "Performance of parameter-estimates in step-stress accelerated life-test with various sample-sizes," IEEE Transactions on Reliability, 51 (3), 2002, pp 271-277.

9. J.R. Van Dorp and T.A. Mazzuchi, "A general Bayes exponential inference model for accelerated life testing," Journal of Statistical Planning and Inference, 119 (1), 2004, pp 55-74.
10. J.R. Van Dorp, T.A. Mazzuchi, G.E. Fornell and L.R. Pollock, "A Bayes Approach to Step-Stress Accelerated Life Testing," , 45 (3), IEEE Transactions on Reliability, 1996, pp 491-498.

\section{BIOGRAPHIES}

J. Rene van Dorp, D.Sc.

Engineering Management and Systems Engineering Depart. The George Washington University

1776 G Street, N.W., Suite 110

e-mail: dorpjr@gwu.edu

J. René van Dorp is an Associate Professor at The George Washington University in Washington DC. Dr. van Dorp received his M.S. (1989) from the Delft University of Technology (The Netherlands) in Mathematics and Computer Science and his D.Sc. (1998) in Operations Research from The George Washington University. His research deals mainly with distribution theory, probabilistic risk assessment and reliability analysis.

T.A. Mazzuchi, D.Sc.

Engineering Management and Systems Engineering Depart.

The George Washington University

1776 G Street, N.W., Suite 110

e-mail:mazzu@gwu.edu

Thomas A. Mazzuchi is Professor and Chair of the Department of Engineering Management and Systems Engineering at the George Washington University, Washington, D.C. Dr. Mazzuchi received a B.A. (1978) in Mathematics from Gettysberg College, Gettysberg, PA, a M.S. (1979) and a D.Sc. (1982), both in Operations Research from the George Washington University, Washington D. C. Dr. Mazzuchi's current research interests include reliability growth assessment, software reliability modeling, design and inference in life testing, reliability estimation as a function of operating environment, maintenance inspection policies, and incorporation of expert judgment into reliability and risk analysis.

Jorge E. Garcidueñas, D.Sc.

Engineering Management and Systems Engineering Depart. The George Washington University

1776 G Street, N.W., Suite 110

e-mail: garyjorg@gwu.edu

Jorge E. Garcidueñas, is a recent graduate from the Engineering Management and Systems Engineering Department at The George Washington University. 